\title{
Commentary
}

\section{Limited Funds for Bladder Cancer Research and What Can We Do About It}

\author{
Joost L. Boormans and Ellen C. Zwarthoff* \\ Erasmus MC Cancer Institute, Departments of Urology and Pathology, Rotterdam, The Netherlands
}

\begin{abstract}
Bladder cancer is frequent, has a high mortality, is expensive and treatment options have not improved over a long time. One of the most important reasons for the relative lack of improvement in treatment of bladder cancer is the low funding for research on bladder cancer. A second reason is that incidence figures in some countries are too low because non-muscle invasive tumors are not registered. We suggest that the bladder cancer community advocates research on bladder cancer and informs policy makers on the underfunding situation. In addition, we need to convince the statisticians that registration should encompass all bladder tumors.
\end{abstract}

Bladder cancer is a very common malignancy with an incidence of 429,800 new cases/year and 165,100 deaths [1]. Despite the high incidence and prevalence, treatment of bladder cancer has not changed much over the past 25 years. Muscle-invasive bladder cancer (MIBC) is treated by radical cystectomy, (neo) adjuvant chemotherapy, or (chemo)radiotherapy [2] whereas non-muscle invasive bladder cancer (NMIBC) is treated by transurethral resection and intravesical therapies [3]. Major breakthroughs in the treatment of NMIBC were the adjuvant instillations with mitomycine $\mathrm{C}$ or related drugs and maintenance therapy with Bacille Calmette-Guérin for high-risk patients to reduce the risk of recurrences. However, after the introduction of these treatment modalities, $>20$ years ago, no new developments or drugs have emerged. In the USA bladder cancer is the 8th (http://seer.cancer.gov) and the UK the 6th most frequent cause of cancer mortality (http://www.cancerresearchuk.org). In 2010, bladder cancer care ranked 9th as the most expensive cancer in the USA with cumulative costs of 4 billion US dollars or $3.2 \%$ of all cancer-related care [4]. In conclusion, $\mathrm{BC}$ is frequent, has a high mortality, is expensive

\footnotetext{
*Correspondence to: Ellen C. Zwarthoff, Erasmus MC Cancer Institute, Departments of Urology and Pathology, P.O. Box 2040, 3000 CA Rotterdam, The Netherlands. Tel.: +31107043929; Fax: +31107044762; E-mail: e.zwarthoff@erasmusmc.nl.
}

and treatment options have not improved over a long time.

In our view, one of the most important reasons for the relative lack of improvement in the treatment of bladder cancer is the low funding for research on bladder cancer. In the USA, research on breast cancer is supported with over 600 million dollars and approximately 300 million is spend on lung, bowel, prostate and leukemia per year. However, research spend on bladder cancer research was a mere 22 million. Figure 1A shows cancer research funding by the National Cancer Institute USA. Data shown are by Carter et al. and date back from 2010 but figures from later years show the same funding trend [5]. Carter et al. presented an exhaustive examination of cancer costs and funding and conclude that bladder and several other cancers are underfunded and suggest to transfer funds towards these cancers. A similar funding discrepancy trend is seen in the UK, based on figures that were extracted from the Cancer Research UK website (Fig. 1B). In 2012 breast, lung and bowel cancer research funding amounted to 31,31 and 30 million pounds, respectively, whereas the amount spend on bladder cancer research was only 4 million pounds. Carter et al. mention in their paper that bladder cancer research in Australia was also underfunded in the years 2003-2005. Unfortunately, we were unable to 

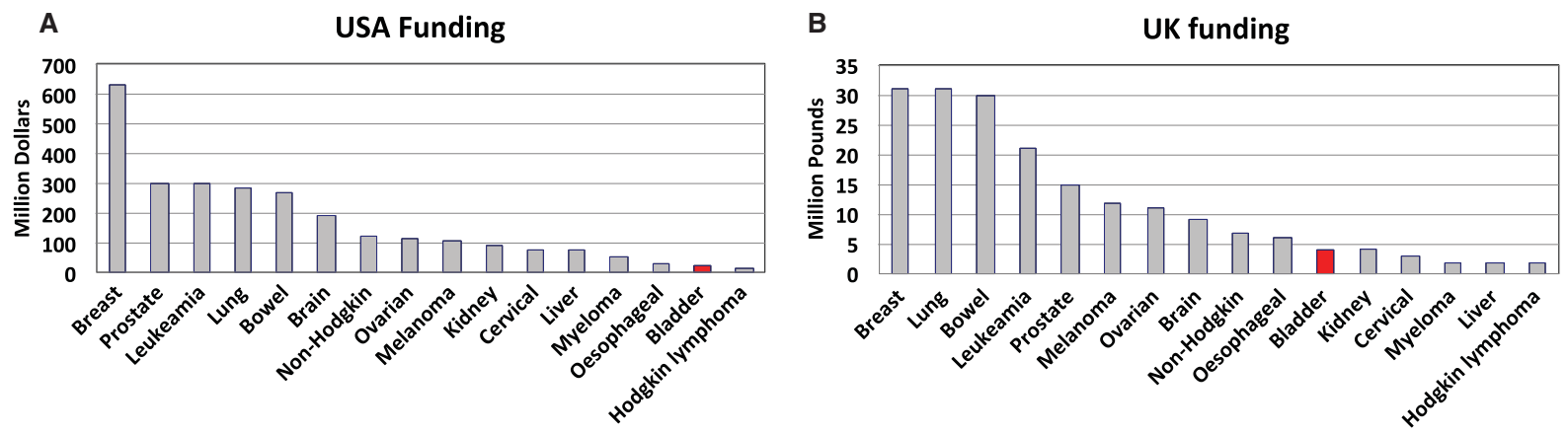

Fig. 1. Cancer research funding for the USA in 2010 (1A) (5) and the UK in 2012 (1B) (http://www.cancerresearchuk.org).
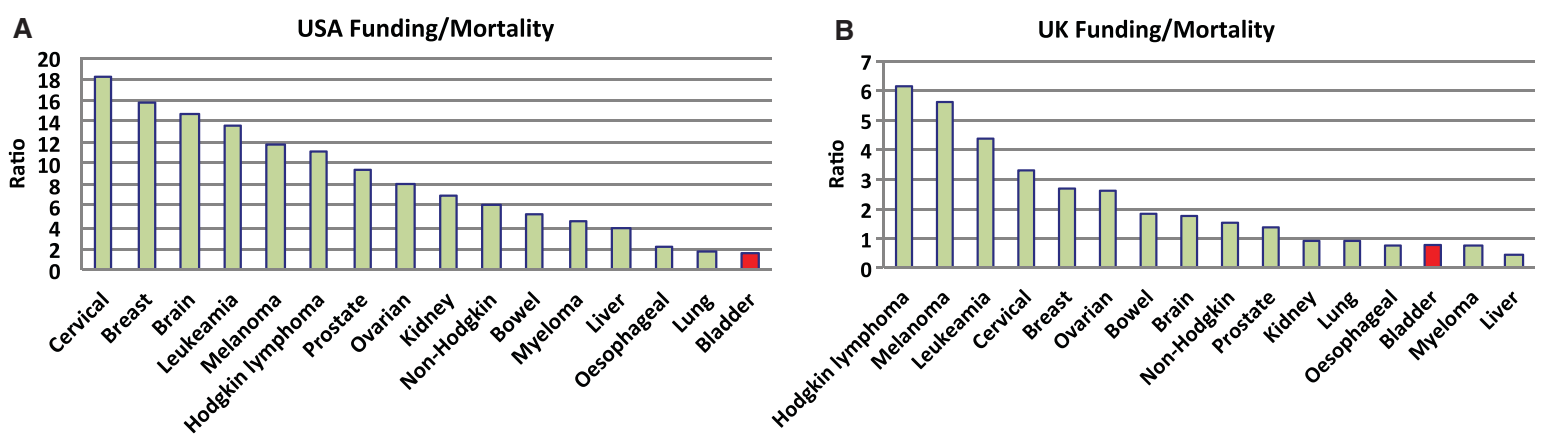

Fig. 2. Cancer research funding divided by cancer specific mortality in the USA (2A) and the UK (2B).

find more recent data. Data for Canada are available from the Canadian Cancer Research Alliance (https://www.ccra-acrc.ca/). In 2013 spend on breast, prostate, colorectal, lung and bladder cancer were 74 , 37, 19, 17 and 2 million Canadian dollars respectively.

It can be argued that cancers that cause more people to die from their disease should receive more research funds. Therefore, we compared research funding per tumor type with cancer-related mortality rates. In Fig. 2, the ratios of cancer funding divided by the number of cancer-specific deaths both in the USA (Fig. 2A) and the UK (Fig. 2B) are shown. We chose mortality rather than incidence because not all countries, for instance the UK, register low-grade noninvasive tumors [1]. Cancer-specific deaths in the USA in 2010 were 40,230 (breast), 157,300 (lung), 51,370 (bowel), 21,840 (leukemia) and 32,050 (prostate) [5]. There were 14,680 patients who died of bladder cancer. Figure 2A clearly shows that bladder cancer is extremely underfunded with respect to the mortality it causes. In the UK 11,716 people died of breast cancer, 16,187 of bowel cancer and there were 5,242 bladder cancer deaths in 2012. As for the USA, the ratio of funding divided by mortality indicates that bladder cancer is underfunded (Fig. 2B). From these figures the picture emerges that most of the cancer research funding in different Western countries goes to those cancer types that are most frequent in incidence. Whether incidence is the sole cause behind this rather uniform outcome is unclear.

A second possible reason for low funding on bladder cancer research might be caused by registration differences. In the Netherlands, bladder cancer mortality is $17 \%$ (1,177 deaths and 6,877 new cases in 2013/2014, http://www.cijfersoverkanker.nl), compared with $22 \%$ in the USA (16,000 deaths and 74,000 cases estimated for 2015, http://seer.cancer.gov) and $50 \%$ in the UK $(5,245$ deaths and 10,705 cases in 2012, http://www.cancerresearchuk.org). Since cancer treatment in these developed countries is expected to be similar, the high mortality figure in the UK suggests that NMIBCs are not registered. This discrepancy was noted by Torre et al. [1] for several countries. Consequently, the incidence figures are lower and it is possible that this may limit awareness of the burden of bladder cancer en hence funding might be affected negatively. NMIBC is very expensive because of the life-long surveillance these patients need [6]. Transurethral resections and cystoscopies together amount to $53 \%$ of all bladder cancer costs in 
Northern European Countries [7]. Of note, in the USA, more cystoscopies are performed than in Europe [8].

We suggest that the bladder cancer community advocates research on bladder cancer and informs policy makers on the underfunding situation. In addition, we need to convince the statisticians that registration should encompass all bladder tumors.

\section{REFERENCES}

[1] Torre LA, Bray F, Siegel RL, Ferlay J, Lortet-Tieulent J, Jemal A. Global cancer statistics, 2012. CA Cancer J Clin 2015;65(2):87-108.

[2] Witjes JA, Comperat E, Cowan NC, De Santis M, Gakis G, Lebret T, Ribal MJ, Van der Heijden AG, Sherif A, European Association of U. EAU guidelines on muscle-invasive and metastatic bladder cancer: Summary of the 2013 guidelines. Eur Urol 2014;65(4):778-92.
[3] Babjuk M, Burger M, Zigeuner R, Shariat SF, van Rhijn BW, Comperat E, Sylvester RJ, Kaasinen E, Bohle A, Palou Redorta J, Roupret M, European Association of U. EAU guidelines on non-muscle-invasive urothelial carcinoma of the bladder: Update 2013. Eur Urol 2013;64(4):639-53.

[4] Mariotto AB, Yabroff KR, Shao Y, Feuer EJ, Brown ML. Projections of the cost of cancer care in the United States: 2010-2020. J Natl Cancer Inst 2011;103(2):117-28.

[5] Carter AJ, Nguyen CN. A comparison of cancer burden and research spending reveals discrepancies in the distribution of research funding. BMC Public Health 2012;12:526.

[6] Botteman MF, Pashos CL, Redaelli A, Laskin B, Hauser R. The health economics of bladder cancer: A comprehensive review of the published literature. Pharmacoeconomics 2003;21(18):1315-30.

[7] Hedelin H, Holmang S, Wiman L. The cost of bladder tumour treatment and follow-up. Scand J Urol Nephrol 2002;36(5):344-7.

[8] Svatek RS, Hollenbeck BK, Holmang S, Lee R, Kim SP, Stenzl A, Lotan Y. The economics of bladder cancer: Costs and considerations of caring for this disease. Eur Urol 2014;66(2):253-62. 